\title{
Black hole masses in active galaxies
}

\author{
Aaron J. Barth ${ }^{1}$ \\ ${ }^{1}$ Astronomy Department, 105-24 Caltech, Pasadena, CA, 91125, USA
}

\begin{abstract}
This contribution reviews two topics of current interest in the study of black hole demographics in active galaxies: Can the stellar velocity dispersions of quasar host galaxies be measured? And can we constrain the black hole mass function below $10^{6} \mathrm{M}_{\odot}$ ?
\end{abstract}

\section{Introduction}

One of the most active areas in observational Active Galactic Nucleus (AGN) research during the past few years has been the development of techniques to estimate the central black hole masses in Seyfert 1 galaxies and quasars. Indirect mass estimators calibrated against the sample of AGNs having masses from reverberation mapping have been applied to a vast range of AGN samples, from local Seyferts to the most distant known quasars. This paper discusses two topics related to these techniques for obtaining masses in broadlined AGNs: the possibility of using host galaxy properties as consistency checks on black hole masses derived from reverberation mapping; and the question of how the black hole mass function can be constrained below $10^{6} \mathrm{M}_{\odot}$ by searching for active galaxies with low-mass black holes.

\section{Can the stellar velocity dispersions of quasar host galaxies be measured?}

The discovery of the $M-\sigma$ correlation (Ferrarese \& Merritt 2000; Gebhardt et al. 2000a) has motivated a great deal of observational effort toward measurement of stellar velocity dispersions in active galaxies. Since the $M-\sigma$ relation is apparently very tight, with $<0.3$ dex scatter (Tremaine et al. 2002), it has been used as a means to estimate black hole masses in AGNs directly from their stellar velocity dispersions. Although this method is indirect, it is still perhaps the best method available for certain classes of AGNs that are not good candidates for reverberation mapping or other more direct techniques, such as radio galaxies (Bettoni et al. 2003; Snellen et al. 2003) or BL Lac objects (Falomo, Kotilainen, \& Treves 2002; Barth, Ho, \& Sargent 2003). Since BL Lac objects have a totally featureless AGN continuum and emission lines are weak or totally absent, detecting the stellar absorption lines in deep ground-based spectra is not difficult, at least for low-redshift objects.

Measurement of stellar velocity dispersions in nearby Seyfert 1 galaxies is usually straightforward as well (e.g., Nelson \& Whittle 1995), except for narrow-line Seyfert 1 galaxies having strong Fe II emission and Ca II emission that tends to fill in the stellar absorption lines. One important application for such measurements is as a consistency check on the black hole masses determined from reverberation mapping of nearby Seyfert 1 galaxies (Wandel, Peterson, \& Malkan 1999; Kaspi et al. 2000). Gebhardt et al. (2000b) and Ferrarese et al. (2001) have shown that the reverberation masses of Seyfert 1 galaxies are in good agreement with the $M-\sigma$ relation of nearby galaxies; this is a reassuring sign that the reverberation measurements give reasonable estimates of the black hole masses. 

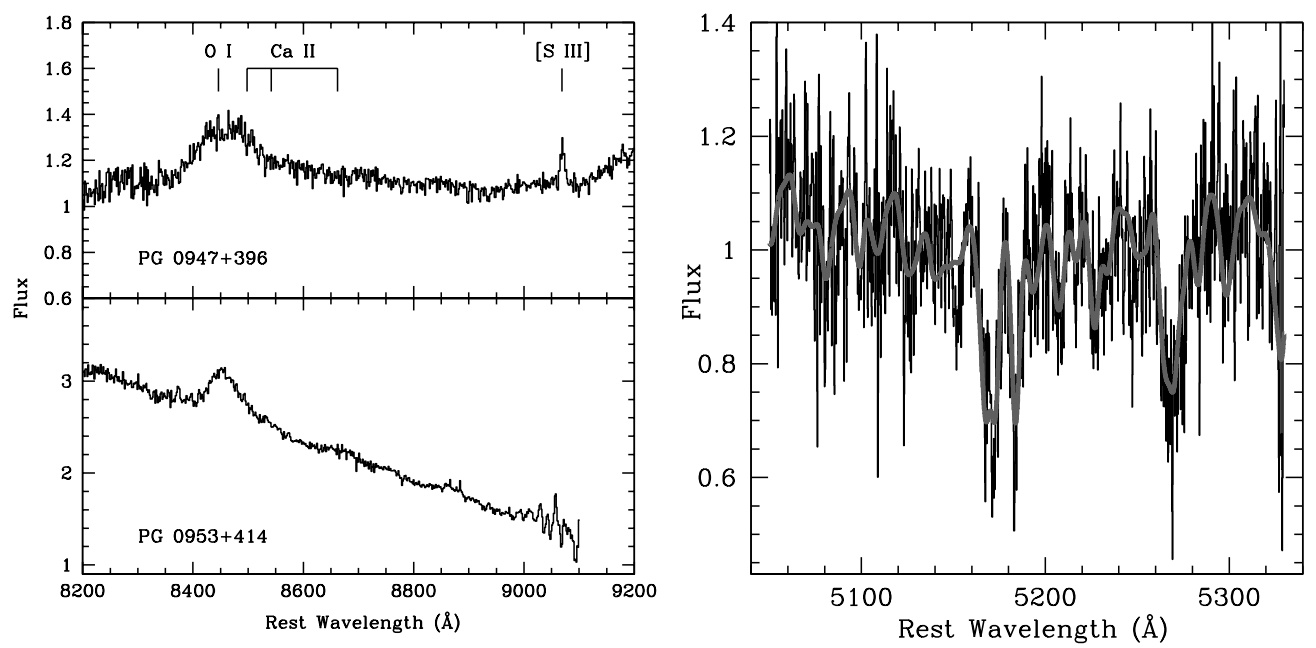

Figure 1. Left panel: Near-infrared spectra of two PG quasars, centered on the Ca II triplet spectral region. The data were obtained with the NIRSPEC spectrograph at Keck. In both cases, the AGN featureless continuum dominates and stellar features are not visible, even though the Ca II lines do not appear in emission. Right panel: Keck spectrum of the host galaxy of the quasar PG $0804+761$, centered on the Mgb lines. The spectrum was obtained with the slit offset by $2^{\prime \prime}$ from the nucleus. Scattered AGN light has been removed by scaling and subtracting the nuclear spectrum. The solid curve overplotted on the spectrum is a $\mathrm{K}$ giant stellar spectrum, broadened by a Gaussian velocity distribution to fit the quasar host spectrum.

Can this consistency check be applied to quasars with black hole masses from reverberation measurements as well? This would be a worthwhile test, since the reverberationmapped Seyferts cover a somewhat limited range in both mass and luminosity. Extending the $M-\sigma$ test to quasars as well would be very worthwhile, since the reverberation sample is one of the fundamental rungs on a "distance ladder" of black hole mass measurement techniques. Nearly all of our understanding of black hole demographics in distant quasars (e.g., Vestergaard 2002; McLure \& Jarvis 2002) relies on this reverberation sample for the basic calibration of the quasar mass scale.

Recently, my collaborators (P. Martini, C. Nelson, L. Ho, J. Greene) and I have attempted to measure the stellar velocity dispersions in a few low-redshift quasars to test whether such observations are feasible. The brute-force approach-directly searching for stellar absorption lines in nuclear spectra- is illustrated in the left panel of Figure 1. For these two quasars, the Ca II triplet lines are redshifted into the near-infrared $J$ band. In the spectra of low-redshift Seyfert 1 galaxies and BL Lac objects the Ca II absorption lines are often visible, albeit diluted substantially by the AGN continuum. The quasars, however, suffer a much greater dilution by the AGN continuum, and we were unable to detect any hint of underlying stellar absorption lines in $\sim 1$ hour exposures at Keck.

The right panel of Figure 1 illustrates an alternative approach which may be useful in some (but not all) cases: in spectra taken off the nucleus, it can be far easier to detect the host galaxy features. In recent years there have been numerous spectroscopic studies of the host galaxies of low-redshift quasars, and it is now a fairly routine matter to detect spectroscopic signatures of the host galaxy stellar population a few arcseconds off the quasar nucleus (e.g., Canalizo \& Stockton 2000; Miller \& Sheinis 2003). Our observations of PG $0804+761$ demonstrate that it is feasible to obtain off-nuclear spectra (offset by $2^{\prime \prime}$ in this case) of sufficient quality to determine the stellar velocity dispersion in the 
off-nuclear light. For this object, preliminary measurements yield a velocity dispersion of $120 \pm 10 \mathrm{~km} \mathrm{~s}^{-1}$ at a projected distance of $3.6 \mathrm{kpc}$ from the nucleus. The main difficulty of this approach is connecting this measurement with the velocity dispersion that would have been obtained in an aperture centered on the nucleus. If the offset slit samples primarily bulge light, it is possible to perform at least a rough extrapolation, but if the aperture samples mainly the light of the host galaxy disk, then the measurement is of little use in determining the position of the quasar on the $M-\sigma$ relation. High-resolution imaging is needed to interpret the results; in this case, HST images have just recently been obtained for this object. This approach, while of limited use at typical ground-based seeing resolution, will benefit greatly from the upcoming deployment of laser guide-star system for adaptive optics on $8-10 \mathrm{~m}$ telescopes. The improvement in spatial resolution will allow observations to be pushed much closer to the nucleus, which will make it much easier to determine the bulge velocity dispersions, at least for objects in which the Ca II triplet lines are redshifted into the observationally accessible ground-based infrared bands.

\section{Can we extend the black hole mass function below $10^{6} \mathbf{M}_{\odot}$ ?}

The fundamental sample that defines our knowledge of black hole demographics consists of galaxies with stellar- and gas-dynamical black hole detections from $H S T$, plus our own Galactic Center and the megamaser galaxy NGC 4258. For this sample, the black hole masses range from about $3 \times 10^{6}$ to $3 \times 10^{9} \mathrm{M}_{\odot}$ and the host galaxy velocity dispersions range from $\sim 65$ to $\sim 400 \mathrm{~km} \mathrm{~s}^{-1}$. Unfortunately, HST does not have high enough spatial resolution to detect black holes below $10^{6} \mathrm{M}_{\odot}$ for distances much beyond the Local Group. At present there is little chance of obtaining dynamical detections of black holes in the mass range $10^{4}-10^{6} \mathrm{M}_{\odot}$ in galaxy centers.

For low-mass galaxies with velocity dispersions below $60 \mathrm{~km} \mathrm{~s}^{-1}$, dynamical searches thus far have led only to upper limits. For M33, analysis of an HST dataset by two independent groups has led to a remarkably tight upper limit of $1500 \mathrm{M}_{\odot}$ (Gebhardt et al. 2001) or $3000 \mathrm{M}_{\odot}$ (Merritt, Ferrarese, \& Joesph 2001). For more distant latetype spirals, the upper limits have been less constraining: one example is IC 342, with $M_{\mathrm{BH}}<5 \times 10^{5} \mathrm{M}_{\odot}$ (Böker, van der Marel, \& Vacca 1999). In a ground-based dynamical study of Virgo cluster dwarf elliptical galaxies, Geha, Guhathakurta, \& van der Marel (2002) determined upper limits of $\sim 10^{6}-10^{7} \mathrm{M}_{\odot}$.

The black hole mass function below $10^{6} \mathrm{M}_{\odot}$ is of particular interest for future gravitywave searches. The space-based interferometer LISA will have good sensitivity for the detection of black hole mergers in the mass range $10^{5}-10^{6} \mathrm{M}_{\odot}$ out to high redshifts (e.g., Hughes et al. 2001), and knowledge of the local black hole mass function in this range would be of use in estimating the expected event rates. The local black hole mass function above $10^{6} \mathrm{M}_{\odot}$ can be determined by combining the bulge luminosity function or velocity dispersion function of galaxies with the $M-\sigma$ relation for black holes, under the assumption that each massive galaxy contains a central black hole (e.g., Yu \& Tremaine 2003; Marconi et al. 2004; Yu \& Lu 2004). The velocity dispersion function of local galaxies (Sheth et al. 2003) is only well-constrained down to $\sim 80 \mathrm{~km} \mathrm{~s}^{-1}$ due to the spectral resolution of the Sloan Digital Sky Survey, and below $10^{7} \mathrm{M}_{\odot}$ there are only a few galaxies with stellar-dynamical black hole detections. Thus, the black hole mass function is not well constrained below this mass range, and the example of M33 suggests that the occupation fraction of black holes in galaxies could be well below unity for galaxies with small $\sigma$. 

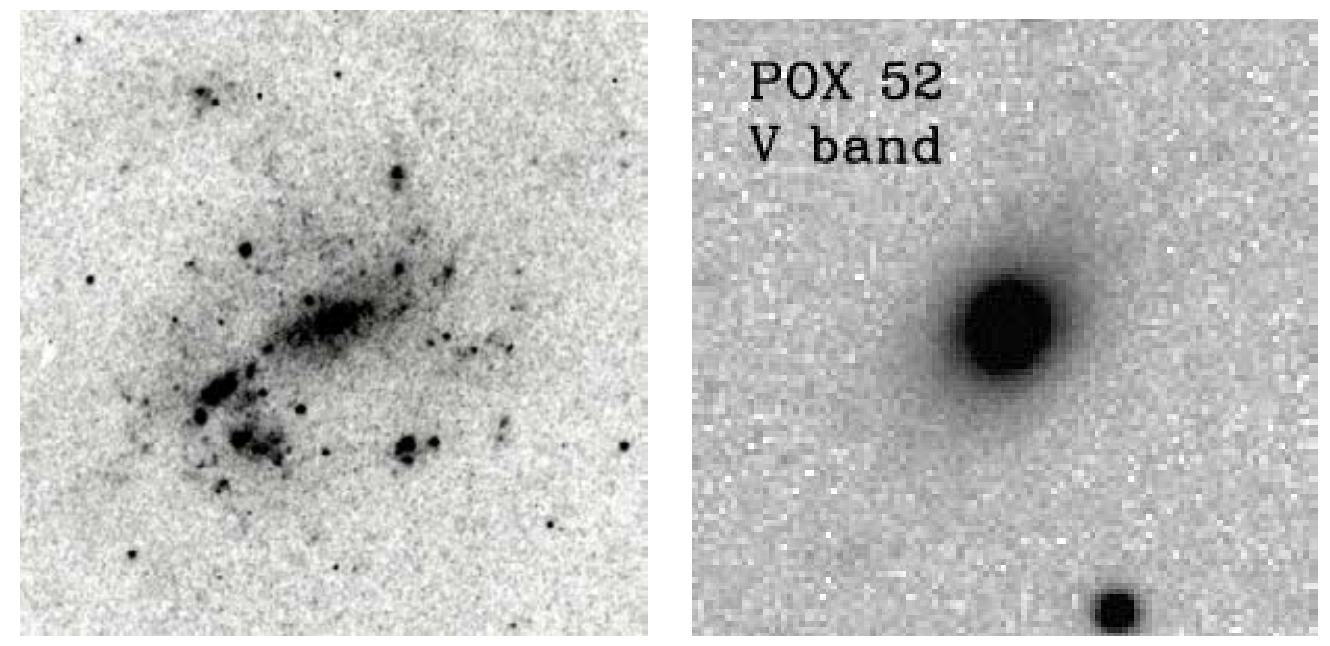

Figure 2. The two best cases of dwarf galaxies harboring active nuclei. Left panel: The late-type spiral galaxy NGC 4395, from the Digitized Sky Survey. Right panel: POX 52, which appears to be a dwarf elliptical, from Barth et al. (2004).

Even if black holes in low-mass galaxies cannot be detected by their dynamical effect on the surrounding stars or gas, at least a fraction of them should be detectable via their accretion luminosity while they are in an active accretion state. For broad-lined AGNs, the black hole masses can then be estimated from the broad-line widths and AGN continuum luminosity (Kaspi et al. 2000). Until recently, however, only two examples of AGNs in dwarf galaxies were known. The best-studied case is NGC 4395, an Sd spiral galaxy at $D \sim 4 \mathrm{Mpc}$, which was first identified as a Seyfert 1 by Filippenko \& Sargent (1989). The nuclear star cluster in NGC 4395 has $\sigma<30 \mathrm{~km} \mathrm{~s}^{-1}$ (Filippenko \& Ho $2003)$, and the black hole mass has been estimated to be in the range $10^{4}$ to a few times $10^{5} \mathrm{M}_{\odot}$ using estimates of the broad-line region radius from photoionization modeling (Kraemer et al. 1999) or from the radius-luminosity correlation for Seyferts (Filippenko \& Ho 2003); and from the detection of a "break" in the power-density spectrum of the X-ray variability (Shih, Iwasawa, \& Fabian 2003).

It is particularly striking that the two best existing data points for the study of black holes in late-type spirals are M33, which does not contain a black hole down to extremely tight limits, and NGC 4395, a galaxy less massive than M33 (it is roughly a magnitude less luminous), which certainly does contain a central black hole, apparently of $10^{4}-10^{5} \mathrm{M}_{\odot}$. The origin of this seeming discrepancy is unclear; perhaps the initial "seed" black holes are ejected from some but not all low-mass galaxies by gravity-wave kicks during mergers (Favata, Hughes, \& Holz 2004; Merritt et al. 2004), or perhaps some disk galaxies never form a seed black hole to begin with. Further progress will require a significant sample of late-type galaxies with either black hole detections or meaningful upper limits to $M_{\mathrm{BH}}$.

The second example is the galaxy POX 52 at $D \approx 90 \mathrm{Mpc}$, discovered in the POX objective-prism search for emission-line objects (Kunth, Sargent, \& Kowal 1981). In a follow-up study, Kunth, Sargent, \& Bothun (1987) called attention to POX 52 as an unusual example of a dwarf galaxy having an AGN spectrum. The object was classified as a Seyfert 2 based on the narrow-line ratios, although they noted that the $\mathrm{H} \beta$ line had a weak broad component with FWHM $\approx 840 \mathrm{~km} \mathrm{~s}^{-1}$. The host galaxy was thought to be a dwarf spiral. More recent observations presented by Barth et al. (2004) show that POX 52 is clearly a Seyfert 1 galaxy, and new imaging data shows the host galaxy to 


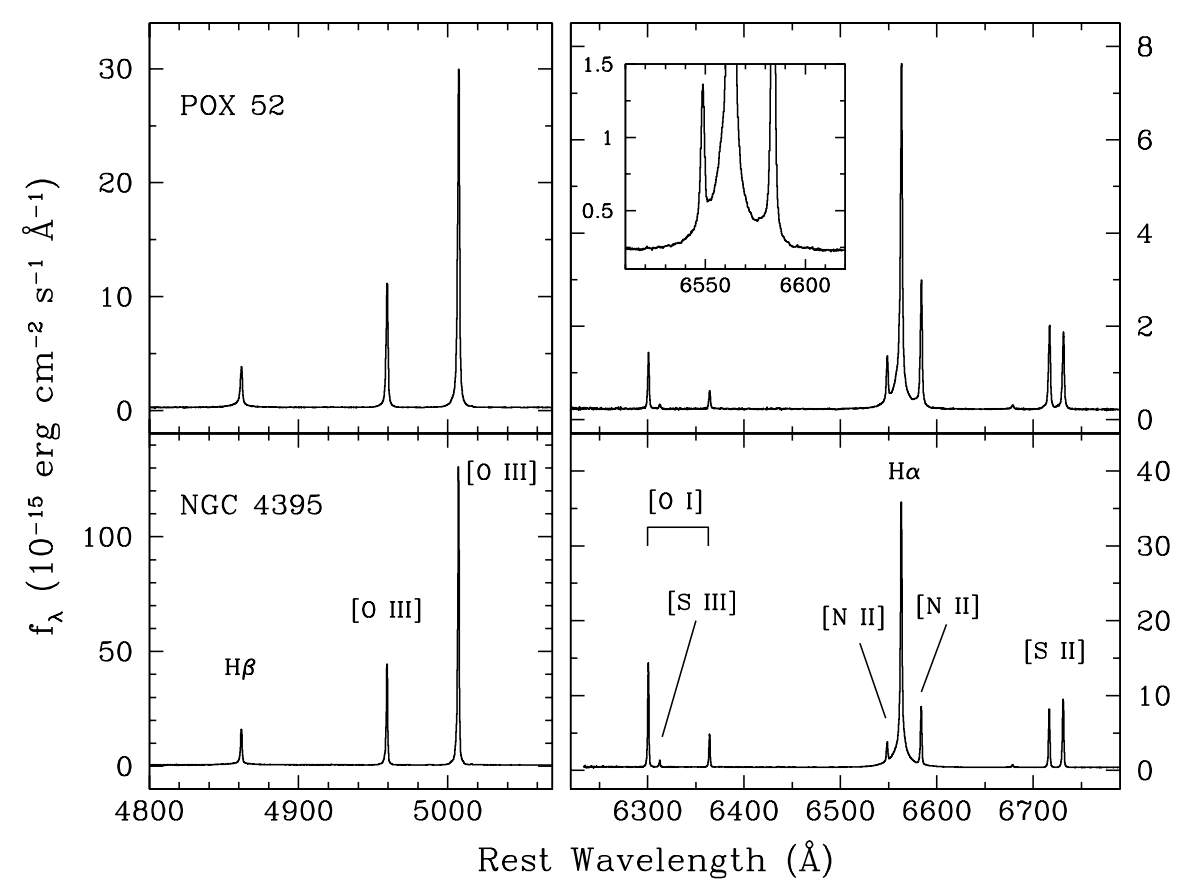

Figure 3. Optical spectra of NGC 4395 and POX 52, from Barth et al. (2004).

be most likely a dwarf elliptical. From $\operatorname{FWHM}(\mathrm{H} \beta)$ and the AGN's optical continuum luminosity, the black hole mass was estimated to be $\sim 1.6 \times 10^{5} \mathrm{M}_{\odot}$, while the stellar velocity dispersion is $36 \pm 5 \mathrm{~km} \mathrm{~s}^{-1}$. With this black hole mass estimate, POX 52 falls almost exactly on an extrapolation of the local $M-\sigma$ relation of Tremaine et al. (2002).

NGC 4395 and POX 52 have remarkably similar optical spectra showing very unusual properties. Both can be classified as narrow-line Seyfert 1 (NLS1) galaxies on the basis of their broad-line widths, but unlike the majority of NLS1s, NGC 4395 and POX 52 have very high-excitation spectra with large $[\mathrm{O}$ III $] / \mathrm{H} \beta$ ratios and high-ionization features such as [Fe VII] $\lambda 5158$ and $\lambda 6086$. They also have extremely narrow forbidden lines $\left(\mathrm{FWHM} \approx 90 \mathrm{~km} \mathrm{~s}^{-1}\right.$ in POX 52), consistent with the low masses and small velocity dispersions of their host galaxies.

The best hope for verification of the black hole mass estimates for these two galaxies would be reverberation mapping to measure the size of their broad-line regions, but it is not yet known whether the emission lines show sufficient variability for reverberation measurements to be successful.

A handful of other AGNs, mostly NLS1s, have black hole mass estimates below $10^{6}$ $\mathrm{M}_{\odot}$ (e.g., Shemmer et al. 2003; Grupe \& Mathur 2004; Botte et al. 2004), but their small numbers and heterogeneous selection makes them of little use in studying the overall demographics of low-mass black holes. To set any meaningful constraints on the occupation fraction of black holes in low-mass galaxies, or on the extension of the $M-\sigma$ relation to low masses, it is necessary to perform a systematic survey for AGNs with low black hole masses. The Sloan Digital Sky survey is an ideal starting point, and an initial sample of 19 Seyfert 1 galaxies with estimated black hole masses below $10^{6} \mathrm{M}_{\odot}$ has recently been reported by Greene \& Ho (2004). While such surveys can only yield a lower limit to the occupation fraction of black holes in low-mass galaxies, this will still 
yield the best constraints that are currently possible on the population of black holes in the mass range below $10^{6} \mathrm{M}_{\odot}$.

\section{Acknowledgements}

I am grateful to Paul Martini, Luis Ho, Charles Nelson, and Jenny Greene for their contributions to the work described here.

\section{References}

Barth, A. J., Ho, L. C., \& Sargent, W. L. W. 2003, ApJ, 583, 134

Barth, A. J., Ho, L. C., Rutledge, R. E., \& Sargent, W. L. W. 2004, ApJ, 607, 90

Bettoni, D., Falomo, R., Fasano, G., \& Govoni, F. 2003, A\&A, 399, 869

Böker, T., van der Marel, R. P., \& Vacca, W. D. 1999, AJ, 118, 831

Botte, V., Ciroi, S., Rafanelli, P., \& Di Mille, F. 2004, AJ, 127, 3168

Canalizo, G., \& Stockton, A. 2000, AJ, 120, 1750

Falomo, R., Kotilainen, J. K., \& Treves, A. 2002, ApJ, 569, L35

Favata, M., Hughes, S. A., \& Holz, D. E. 2004, ApJ, 607, L5

Ferrarese, L., \& Merritt, D. 2000, ApJ, 539, L9

Ferrarese, L., Pogge, R. W., Peterson, B. M., Merritt, D., Wandel, A., \& Joseph, C. L. 2001, ApJ, 555, L79

Filippenko, A. V., \& Ho, L. C. 2003, ApJ, 588, L13

Filippenko, A. V., \& Sargent, W. L. W. 1989, ApJ, 342, L11

Gebhardt, K., et al. 2000a, ApJ, 539, L13

Gebhardt, K., et al. 2000b, ApJ, 543, L5

Gebhardt, K., et al. 2001, AJ, 122, 2469

Gebhardt, K., et al. 2003, ApJ, 583, 92

Geha, M., Guhathakurta, P., \& van der Marel, R. P. 2002, AJ, 124, 3073

Greene, J. E., \& Ho, L. C. 2004, ApJ, in press

Grupe, D., \& Mathur, S. 2004, ApJ, 606, L41

Hughes, S. A., Márka, S., Bender, P. L., \& Hogan, C. J. 2001, in Proceedings of the APS/DPF/DPB Summer Study on the Future of Particle Physics (Snowmass 2001), (ed. N. Graf), eConf C010630

Kaspi, S., Smith, P. S., Netzer, H., Maoz, D., Jannuzi, B. T., \& Giveon, U. 2000, ApJ, 533, 631

Kraemer, S. B., Ho, L. C., Crenshaw, D. M., Shields, J. C., \& Filippenko, A. V. 1999, ApJ, 520, 564

Kunth, D., Sargent, W. L. W., \& Bothun, G. D. 1987, AJ, 93, 29

Kunth, D., Sargent, W. L. W., \& Kowal, C. 1981, A\&AS, 44, 229

Marconi, A., Risaliti, G., Gilli, R., Hunt, L. K., Maiolino, R., \& Salvati, M. 2004, MNRAS, 351, 169

McLure, R. J., \& Jarvis, M. J. 2002, MNRAS, 337, 109

Merritt, D., Milosavljević, M., Favata, M., Hughes, S. A., \& Holz, D. E. 2004, ApJ, 607, L9

Merritt, D., Ferrarese, L., \& Joseph, C. L. 2001, Science, 293, 1116

Miller, J. S., \& Sheinis, A. I. 2003, ApJ, 588, L9

Nelson, C. H., \& Whittle, M. 1995, ApJS, 99, 67

Shemmer, O., Uttley, P., Netzer, H., \& McHardy, I. M. 2003, MNRAS, 343, 1341

Sheth, R. K., et al. 2003, ApJ, 594, 225

Shih, D. C., Iwasawa, K., \& Fabian, A. C. 2003, MNRAS, 341, 973

Snellen, I. A. G., Lehnert, M. D., Bremer, M. N., \& Schilizzi, R. T. 2003, MNRAS, 342, 889

Tremaine, S., et al. 2002, ApJ, 574, 740

Vestergaard, M. 2002, ApJ, 571, 733

Wandel, A., Peterson, B. M., \& Malkan, M. A. 1999, ApJ, 526, 579

Yu, Q., \& Lu, Y. 2004, ApJ, 602, 603

Yu, Q., \& Tremaine, S. 2002, MNRAS, 335, 965 